\title{
Response latency, confidence, and ROCs in auditory signal detection*
}

\author{
DAVID S. EMMERICH† and JAMES L. GRAY \\ State University of New York at Stony Brook, Stony Brook, New York 11790 \\ and \\ CHARLES S. WATSON and DAVID C. TANIS \\ Central Institute for the Deaf, St. Louis, Missouri 63110
}

Response latencies were obtained from $10 \mathrm{Ss}$ in auditory signal-detection experiments. The response latencies were inversely related to certainty that a signal was (or was not) presented. The S's decision criterion was found to have an influence on response latency, which was consistent with the hypothesis that stimuli close to the current criterion elicit longer response latencies than stimuli more distant from the criterion. Comparisons among receiver operating characteristics derived from binary decisions, from the latencies of binary decisions, and from confidence ratings show that response latencies and binary decisions together yield more information about the stimulus than does the binary decision alone. However, the increment in information gained from the measurement of response latencies is in general (though not for every $\mathbf{S}$ ) smaller than that gained by shifting from yes-no responses to a confidence-rating procedure.

In an experiment in which Ss were asked to judge which of two lines was longer, Johnson (1939) recorded both the length of time necessary to make these judgments and Ss' ratings of confidence that their judgments were correct. For differences in the lengths of the lines which exceeded a certain amount, the Ss' judgments were correct $100 \%$ of the time. Differences just large enough to yield judgments which were always correct, however, were still too small to give the Ss great confidence in their responses. For increasingly greater differences, Ss' confidence increased until they eventually used the confidence ratings associated with "maximum certainty." The time required to make the judgments of length decreased as the percentage of correct responses varied from near chance to near perfect. This response latency continued to decrease over the range of differences associated with moderate-to-complete confidence in the judgments, and it decreased even more over the range of differences larger than those which led to maximum-certainty responses.

*Experiments 1 and 2 were supported in part by the Research. Foundation of the State of New York. Experiment 3 was supported by PHS Research Grant NS 03856 (to the Central Institute for the Deaf) from the National Institute of Neurological Diseases and Stroke of the National Institutes of Health. D.C.T. is a predoctoral fellow supported by PHS Grant GM 1900 to the Department of Psychology, Washington University, St. Louis, Missouri. tRequests for reprints should be sent to David S. Emmerich, Department of Psychology, State University of New York at Stony Brook, Stony Brook, New York 11790.
Thus, as Woodworth and Schlosberg (1954) commented, response latency, in Johnson's study, could be used to scale the effects of stimulus differences over a broader range of values than could either binary decisions or confidence ratings. Many other Es have subsequently investigated the interrelations among judgmental latency, stimulus magnitudes, confidence ratings, and accuracy. Festinger (1943a, b), and Medin, Borkhius, and Davis (1970), among others, have contributed to the conception of an inverse relationship between the difficulty of a decision in a psychophysical experiment and the time required to make it.

Gescheider, Wright, Weber, Kirchner, and Milligan (1969) suggested that response latency in psychophysical experiments is not determined solely by stimulus magnitude, but rather by the relation between stimulus magnitude and the decision criterion held by the observer. Cartwright (1941) had reached a similar conclusion on the basis of the results of a variety of experiments in which several categories of response were employed, summing up his results with the suggestion that "the time required for deciding to which category a stimulus belongs increases as the stimulus approaches the border of a range of equivalent stimuli [p. 195]."

Gescheider et al (1969) found, in an experiment on the detectability of vibrotactile signals, that responses with lower probabilities of occurrence tended to have longer latencies. Response probabilities were manipulated by changing the a priori probability of signal presentation. It was predicted that when the inputs leading to a given response were close to the criterion for that response, along some decision axis, the latencies for that response would be unusually long. The data supported this prediction, showing long latencies, for example, for "yes" responses under a strict decision criterion and for "no" responses under a lax decision criterion. These results are consistent with those of earlier studies (Fernberger, Glass, Hoffman, \& Willig, 1934; Festinger, 1943a) in which the Ss' decision criteria were varied by instruction.

Gescheider et al discuss their results in terms of the theory of signal detectability (TSD), a theory which emphasizes the importance of the S's decision criterion in psychophysical experiments (see Swets, Tanner, \& Birdsall, 1961). In this theory, the receiver operating characteristic (ROC) assumes great importance. The ROC is often used to make inferences about the forms of distributions upon which, in TSD, the $\mathrm{S}$ is assumed to base his responses. Carterette, Friedman, and Cosmides (1965) and Norman and Wickelgren (1969) have each proposed their own method of deriving empirical ROCs from distributions of response latencies. David $M$. Green (personal communication, 1970) points out that there is a simple relationship between these two methods of deriving ROCs from latency distributions.

The experiments reported here are a continuation of the above line of investigation. Their particular goals were to determine the relations among response latencies for various decision criteria in an auditory detection task, and to compare ROCs constructed from binary decisions, confidence ratings, and response latencies. Experiments 1 and 2 were conducted

Table 1

Means (in Milliseconds) and Standard Deviations (in Parentheses) of Latencies of $Y$ es-No Responses on Trials on Which the Indicated Rating Responses Were Made (Experiment 1)

\begin{tabular}{|c|c|c|c|c|c|c|}
\hline & $\begin{array}{c}\text { Rating 1: } \\
\text { Very Sure } \\
\text { Yes }\end{array}$ & $\begin{array}{l}\text { Rating 2: } \\
\text { Moderately } \\
\text { Sure Yes }\end{array}$ & $\begin{array}{l}\text { Rating 3: } \\
\text { Somewhat } \\
\text { Sure Yes }\end{array}$ & $\begin{array}{c}\text { Rating 4: } \\
\text { Somewhat } \\
\text { Sure No }\end{array}$ & $\begin{array}{l}\text { Rating 5: } \\
\text { Moderately } \\
\text { Sure No }\end{array}$ & $\begin{array}{c}\text { Rating 6: } \\
\text { Very Sure } \\
\text { No }\end{array}$ \\
\hline $\begin{array}{l}\text { S } 1 \\
\text { S } 2 \\
\text { S } 3\end{array}$ & $\begin{array}{l}541(176) \\
554(109) \\
591 \quad(78)\end{array}$ & $\begin{array}{ll}631 & (214) \\
611 & (132) \\
616 & (119)\end{array}$ & $\begin{array}{ll}818 & (218) \\
699 & (139) \\
840 & (222)\end{array}$ & $\begin{array}{ll}874 & (206) \\
768 & (129) \\
956 & (219)\end{array}$ & $\begin{array}{l}704(198) \\
714(115) \\
810(160)\end{array}$ & $\begin{array}{l}640(118) \\
697(114) \\
804(123)\end{array}$ \\
\hline
\end{tabular}


Table 2

Means (in Milliseconds) and Standard Deviations (in Parentheses) of Latencies of Yes and No Responses (Experiment 1)

\begin{tabular}{lccccccc}
\hline & $\begin{array}{c}\text { Yes Given } \\
\text { Signal }\end{array}$ & $\begin{array}{c}\text { Yes Given } \\
\text { Noise }\end{array}$ & $\begin{array}{c}\text { All Yes } \\
\text { Responses }\end{array}$ & $\begin{array}{c}\text { No Given } \\
\text { Signal }\end{array}$ & $\begin{array}{c}\text { No Given } \\
\text { Noise }\end{array}$ & $\begin{array}{c}\text { All No } \\
\text { Responses }\end{array}$ \\
\hline S 1 & $619(213)$ & $823(254)$ & $650(231)$ & $768(239)$ & $695(182)$ & $708(194)$ \\
S 2 & $620(133)$ & $706(158)$ & $639(144)$ & $731(122)$ & $715(121)$ & $719(121)$ \\
S 3 & $730(207)$ & $949(208)$ & $762(221)$ & $952(209)$ & $\mathbf{8 6 7}(198)$ & $884(203)$ \\
\hline
\end{tabular}

at the State University of New York at Stony Brook; Experiment 3 was conducted independently at the Central Institute for the Deaf.

\section{EXPERIMENT 1}

Experiment 1 was conducted in order to learn whether the latency of "yes" and "no" responses in a single-interval detection experiment could be reasonably interpreted as a measure of confidence. As in Johnson's early experiment, the listeners made a binary decision and then a confidence rating on each trial.

\section{Method}

Subjects. The Ss were three male college students at the State University of New York at Stony Brook. All Ss had clinically normal hearing and none had previously served as a $S$ in an auditory experiment. The $\mathrm{Ss}$ were paid for their participation.

Apparatus. Acoustic stimuli were produced by means of standard laboratory components, including an oscillator, noise generator, electronic switch, and interval timer. The stimuli were presented binaurally and in phase by means of earphones (Telephonics, Model TDH-39). Response latencies were measured with an electronic counter. The Ss were tested individually in a sound-attenuating chamber.

Procedure. The task was to detect 400-Hz tone pulses, $200 \mathrm{msec}$ in duration, presented in a background of continuous white noise. The tone pulses were gated at zero crossings in order to reduce switching transients. The noise was band-limited by the earphone response and had a spectral level, $\mathrm{N}_{\mathrm{o}}$, of approximately $45 \mathrm{~dB}$ SPL. On each trial an observation light flashed on for $200 \mathrm{msec}$, and, with probability 0.5 , a tone pulse was presented accompanying the light. During the first one and one-half sessions of the experiment, the Ss responded simply by pushing a "yes" or "no" button following the observation interval to indicate their decision as to whether a signal had been presented or not. In later sessions, this binary decision was followed by a confidence rating which could indicate any of six categories of confidence, ranging from high confidence that the signal had been presented to high confidence that it had not been presented. When only yes-no responses were made, each trial consisted of a 200-msec warning light, a $500-\mathrm{msec}$ pause, a 200-msec observation interval light, a $1,500-\mathrm{msec}$ response interval, a 200-msec pause, a 200-msec feedback interval, and a final $750-\mathrm{msec}$ pause. During the feedback interval a light was illuminated if a signal had been presented on that trial. When both yes-no responses and ratings were made on the same trial, the trial structure remained the same except that following the yes-no response the trial was interrupted and would not progress until the rating response was made.

Experimental sessions were divided into halves separated by a rest break of 5-10 min. Each half of a session consisted of a 20-trial practice block followed by three 60-trial data blocks. These blocks of trials were separated by short rest periods, and each block was preceded by demonstrations of the signal both at a level $10 \mathrm{~dB}$ greater than and at the same level as that used during the blocks of trials.

The signal energy, E, was adjusted so that each $S$ could achieve $75 \%-85 \%$ correct responses with a medium decision criterion $\left(10 \log E / N_{0}\right.$ was 11 for $S 1,10$ for $S 2$, and 11 for $S 3$ ). The Ss were informed that the a priori probability of signal presentation was 0.5 and that they would get the highest percent correct if they responded "yes" and "no" about equally often. No other attempt was made to influence the $\mathrm{Ss}^{\prime}$ response criteria.

Without the Ss' knowledge, the latencies of their yes-no responses were recorded (to the nearest millisecond) from the beginning of the observation interval. No pressures were put on the Ss to respond rapidly, other than explaining that the yes-no response had to be completed during the 1,500-msec response interval. The yes-no responses were made by pressing one of two buttons which were mounted $3 \mathrm{in}$. from the bottom of the sloping front panel $(9 \times 12$ in.) of a simple box, and centered 3 in. apart. The left button was labeled "yes" and the right "no." The Ss were told to keep a thumb or finger on each of these buttons at all times except when making a rating. It was observed that Ss complied with this request.
The Ss made rating responses by pressing one of six buttons mounted in a horizontal row $2 \frac{1}{2}$ in. above the yes-no buttons.

The experiment consisted of one and one-half sessions of practice on the yes-no task, one and one-half sessions of practice with both yes-no and rating responses, and three sessions of actual data collection with both yes-no and rating responses.

\section{Results and Discussion}

The mean latencies of the initial yes-no responses for all trials (whether or not a signal was presented) on which a given rating response was made are presented in Table 1. As the standard deviations of these latencies indicate, there is considerable overlap in the latency distributions associated with the different rating responses. Although the Ss were not specifically instructed to press one of the left three rating buttons (labeled "very sure yes," "somewhat sure yes," and "moderately sure yes") following an initial "yes" response, and one of the correspondingly labeled three buttons on the right following an initial "no" response, no $S$ ever changed his mind between the binary decision and the rating.

Table 2 presents the means and standard deviations of the latencies of all "yes" and all "no" responses and of these responses partitioned in terms of the presence or absence of the signal.

Gescheider et al (1969) hypothesize that the further the representation of the stimulus from the response criterion on the decision axis assumed in TSD, the shorter the response latency. Consistent with this hypothesis is the fact that in Table 1 the data of each $S$ show the mean latencies of initial "yes" responses to be smaller the greater the confidence that a signal was presented, and the mean latencies of initial "no" responses to be smaller the greater the confidence that noise alone was presented. Similarly, the Pearson product-moment correlation coefficients between the latencies of "yes" responses and confidence ratings made on individual trials for the three Ss are $.48, .42$, and .47 , and those between the latencies of "no" responses and confidence ratings are $-.30,-.22$, and -.33. Katz $(1970)$ has recently found response latency to be inversely related to $S$ 's confidence in the correctness of their responses in a situation involving same-different judgments.

The fact that, as can be seen from Table 2, correct responses are faster than incorrect responses is consistent with the Gescheider et al hypothesis, since (for a medium criterion placement) values leading to a correct 


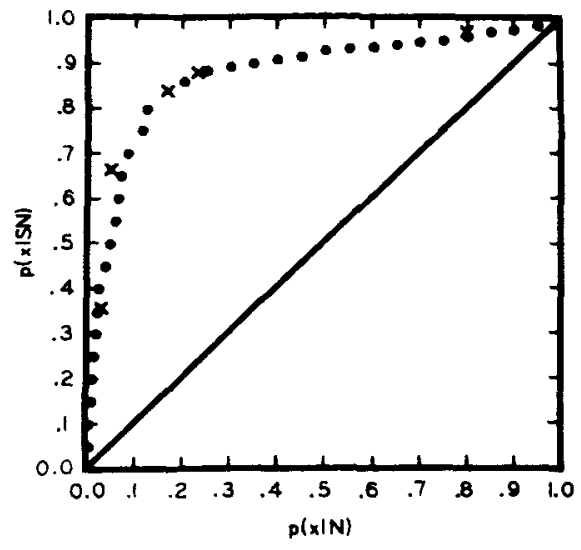

Fig. 1. Rating-scale and latency ROCs obtained from $S 1$. The Xs are from ratings and the circles are derived from response latencies.

response will be further from the criterion, on the average, than those leading to an incorrect response. If, as a number of authors propose (see Green \& Swets, 1966, pp. 95-116), it is also hypothesized that there is greater variance in the signal-plus-noise (SN) distribution than in the noise (N) distribution on the decision axis, other aspects of the data of Tables 1 and 2 can be predicted. Thus, the facts that for each $S$ the range of mean latencies of "yes" responses in Table 1 is greater than that of "no" responses, and that, as Table 2 shows, "yes" responses are faster than "no" responses, would both follow (for a medium criterion placement) from the additional assumption that the SN distribution is less concentrated about its mean than is the $\mathrm{N}$ distribution.

The rating responses of the Ss were analyzed using a method employed by several earlier investigators (such as Egan, Schulman, \& Greenberg, 1959; Swets et al, 1961; Watson, Rilling, \& Bourbon, 1964), in order to obtain the ROCs represented by the $X s$ in Figs. 1-3. Briefly, these Xs represent the cumulative proportions of $\mathrm{SN}$ trials on which ratings indicating a given degree (or greater) of certainty that a signal was presented were made, plotted against the same proportions of $\mathrm{N}$ trials.

The filled circles in Figs. 1-3 were obtained by treating the latencies of yes-no responses as being analogous to confidence ratings. "Yes" responses of short latency were treated as rating responses indicating that the $S$ was very sure that a signal had been presented. "Yes" responses of progressively longer latency were considered to correspond to ratings indicating progressively less certainty that a signal had been presented. Long-latency "no" responses were then taken to indicate still less certainty and short-latency "no" responses the least certainty of all that a signal had been presented. The data of Table 1 suggest that this assumption is not unreasonable for the present experiment. After assuming this correspondence, the filled circles in Figs. 1-3 were obtained by the procedure used to determine the Xs. After the "yes" and "no" responses were appropriately ordered in terms of their latencies, the "rating categories" were determined by dividing up the latency distributions of these responses. Norman and Wickelgren (1969) have generated ROCs from response latencies in this fashion in an investigation of short-term memory. When ROCs are derived from confidence ratings, the spacing of the points is fixed by the S's use of the rating categories. The spacing of the points on ROCs derived from latency distributions is, however, largely under the E's control since the E determines the increments in latency which are used to generate the points.

No S ever responded "yes" and then made a rating indicating a decision that no signal had been presented, or responded "no" and then made a rating indicating that a signal had been presented. This being the case, as the discussion by Norman and Wickelgren (1969) makes clear, the third point on a given rating $R O C$ in Figs. 1-3 must also fall on the ROC derived from response latencies. This is true since the third point on such a rating $R O C$ is obtained by cumulating all of the "yes ratings" (separately for $\mathrm{SN}$ and $\mathrm{N}$ trials), and this must also be the point which is obtained by cumulating all of the initial "yes" responses meeting successive latency criteria.

The other points on the rating ROCs of Figs. 1-3 fall very close to the points derived from response latencies. However, this observation must be considered with some caution for two

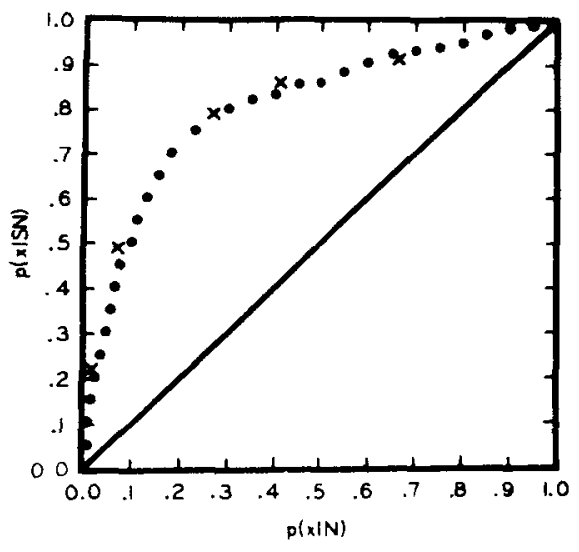

Fig. 2. ROCs obtained from $\mathbf{S}$. (See caption for Fig. 1.)

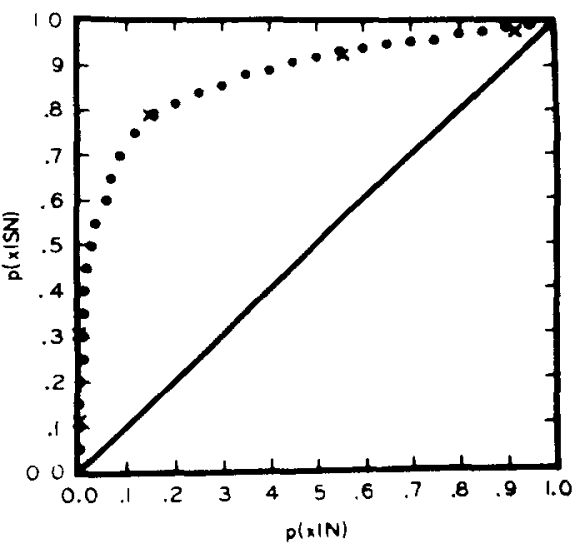

Fig. 3. ROCs obtained from $\mathbf{S} 3$. (See caption for Fig. 1.)

reasons. First, since the $S$ s could remember their yes-no responses while making their rating responses, the two sorts of responses were presumably not made independently. Second, due to the way in which the latency-based ROCs were constructed, they cannot depart too radically from the rating ROCs. The upper portion of the latency-based ROC must fall in the rectangle with the line connecting the binary-decision operating point, $(p(y e s \mid N), p(y e s \mid S N))$, and the point $(1,1)$ for a diagonal, and with sides parallel to the axes of the ROC coordinate system. Similarly, the lower portion must fall in the rectangle with sides parallel to these axes, and the line connecting the origin and the binary-decision operating point for a diagonal. Furthermore, since previous research (e.g., Carterette et al, 1965; Gescheider et al, 1969) indicated that in the yes-no signal-detection situation correct responses are faster than incorrect ones, it would have been surprising if the latency-based ROCs in the present experiment had fallen below the just-mentioned diagonals of these rectangles. The fact that the latency-based ROCs fall above this pair of straight lines does indicate that a better a posteriori prediction as to whether or not a signal had been presented could be made, however, by considering the binary decision and its latency, than by considering only the binary decision. It is exactly this type of additional information that is provided by confidence ratings.

That portion of each latency ROC in Figs. 1-3 which is based upon yes responses (the filled circles between the origin and the third $X$ ) exhibits greater curvature than does the remainder of the ROC. This suggests that the processes which lead to "no" responses may differ in some ways from those which lead to "yes" responses. The fact that the points 


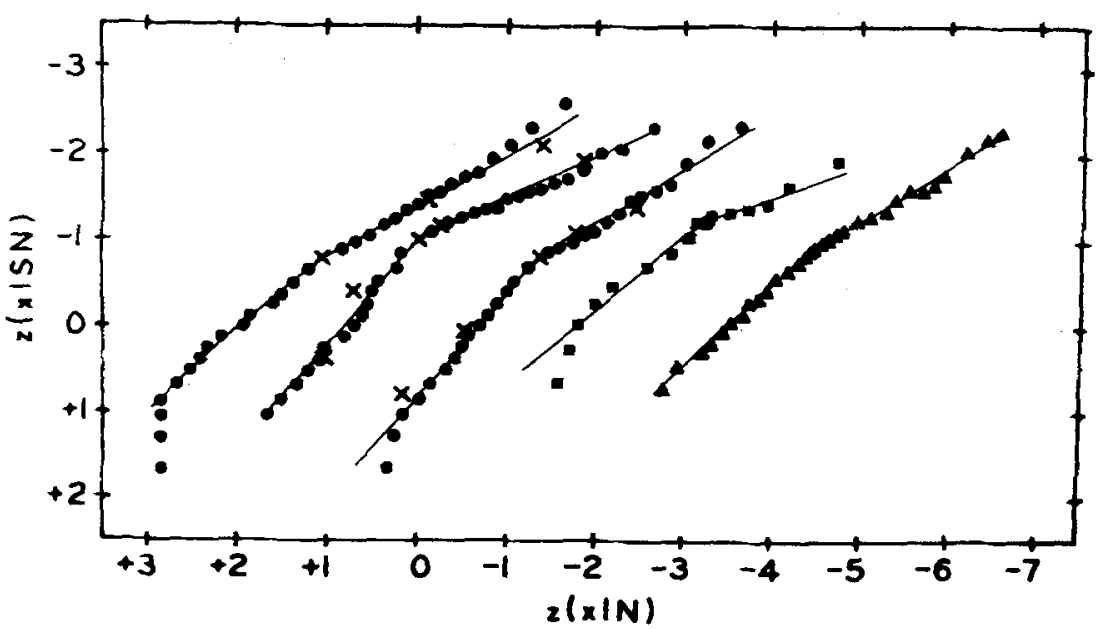

Fig. 4. ROCs plotted on normal-normal coordinates, for results of Experiment 1. The first three ROCs, reading from left to right, are replotted from Figs. 3, 1, and 2, respectively. The squares represent data from Emmerich (1968), and the triangles represent data from Watson et al (1964). Points on the five curves have been displaced to the right by $0,1,2,4$, and 5 units, respectively. Data from the earlier experiments are included because of the similarity of the "elbows" near the negative diagonal to those "elbows" discernible in the latency ROCs from Experiment 1. which were derived from rating responses lie close to the latency ROCs further suggests that such differences in curvature are not solely due to the fact that the ROCs in question were derived from response latencies.

Figure 4 presents the ROCs of Figs. 1-3 plotted on normal-normal coordinates. In this coordinate system ROCs generated, as in TSD, from over-lapping normal distributions on the decision axis are straight lines. The ROCs in Fig. 4 which are based on response latency do not appear to be straight lines, but then neither do those based on confidence ratings. Figure 4 also includes ROCs based on mechanical devices with continuous ranges) from some earlier experiments (Emmerich, 1968; Watson et al, 1964). Both the ROCs from the present experiment and the earlier ones from rating experiments can be fitted by two straight lines intersecting in the neighborhood of the negative diagonal (the line for which $p$ (yes $\mid \mathrm{SN})=$ $1-p($ yes $! N))$, or in the case of the 1 atency-based ROCs, in the neighborhood of the yes-no operating point. The angle between the two great, however, and presumably would be easily overlooked if only a few points were obtained on an empirical ROC. The departure of the form of the ROCs presented in Fig. 4 from that predicted by the overlapping-normal-distribution models of TSD is discussed in the last section of this paper.

\section{EXPERIMENT 2}

In Experiment 2 the Ss were confidence ratings (made using branches of these ROCs is not very instructed to vary their response criteria in a yes-no signal-detection situation rather than being allowed to adopt a medium criterion as in Experiment 1. Response latencies were measured for each response.

\section{Method}

Subjects. The Ss were college students (two males and one female) at the State University of New York at Stony Brook, each of whom had clinically normal hearing. One of the Ss (S 1) had had previous experience in an auditory experiment. The Ss were paid for their participation.

Apparatus. The apparatus was the same as that employed in Experiment 1, except that there were no rating buttons on the box on which the yes-no response buttons and indicator lights were mounted.

Procedure, The procedure was similar to that employed in the initial one and one-half sessions of Experiment 1 when $S s$ received practice on the simple yes-no signal-detection task, except that in Experiment $\mathbf{Z}$ the $\mathbf{S}$ was asked to vary his response criterion from time to time, and the number of trials in a data block was reduced from 60 to 50 . The Ss were trained to adopt different criteria by instructing them to vary the proportion of trials on which they made "yes" responses. In practice sessions they were told whether or not they were saying "yes" on an appropriate proportion of the trials, and were encouraged to modify their performance if they were not. No attempts to change a given criterion were made after the practice sessions with that criterion were completed. Three to five practice sessions were given with each criterion, followed by five sessions in which experimental data were collected.

The Ss were asked to adopt three response criteria: a strict, a medium, and a lax criterion. For $S 1$ it was decided that the strict criterion should lead to a "yes" response on approximately $5 \%$ of the signal trials, and the lax criterion should lead to a "yes" response on approximately $90 \%$ of the signal trials. In order to investigate a wide range of decision criteria, S 2 and $\mathrm{S} 3$ were allowed to adopt less extreme strict and lax criteria. For all Ss the medium criterion was the one which the $S$ selected as being most natural. The sequence of criteria for $S 1$ was medium, lax, strict; for $\mathbf{S} 2$ it was lax, strict, medium; and for $\mathrm{S} 3$ it was medium, lax. Due to unforeseen problems, it was impossible to test S 3 under the third criterion. Values of $10 \log \left(E / N_{0}\right)$ were 12 for $S 1,11$ for $S 2$, and 11 for $S 3$.

Results and Discussion

The average latencies of "yes" and "no" responses in the various conditions of Experiment 2 are presented in Table 3. The data of $S 1$ are quite orderly with respect to criterion placement: as a given response was made more frequently, its mean latency decreased. Carterette et al (1965) and Gescheider et al (1969) found this relationship to hold, though there were exceptions to the general trend (in particular, see Table 1 of Carterette et al, 1965). This result is understandable in terms of the
Table 3

Mean Latenctes of $Y$ es and No Responses in Milliseconds (Experiment 2)

\begin{tabular}{|c|c|c|c|c|c|c|c|}
\hline & Criterion & $\begin{array}{c}\text { Yes } \\
\text { Given } \\
\text { Signal }\end{array}$ & $\begin{array}{l}\text { Yes } \\
\text { Given } \\
\text { Noise }\end{array}$ & $\begin{array}{c}\text { All } \\
\text { Yes } \\
\text { Responses }\end{array}$ & $\begin{array}{c}\text { No } \\
\text { Given } \\
\text { Signal }\end{array}$ & $\begin{array}{c}\text { No } \\
\text { Given } \\
\text { Noise }\end{array}$ & $\begin{array}{c}\text { All } \\
\text { No } \\
\text { Responses }\end{array}$ \\
\hline S 1 & $\begin{array}{l}\text { Lax } \\
\text { Medium } \\
\text { Strict }\end{array}$ & $\begin{array}{l}671 \\
738 \\
827\end{array}$ & $\begin{array}{l}729 \\
830 \\
923\end{array}$ & $\begin{array}{l}695 \\
752 \\
836\end{array}$ & $\begin{array}{l}841 \\
785 \\
752\end{array}$ & $\begin{array}{l}838 \\
775 \\
736\end{array}$ & $\begin{array}{l}839 \\
777 \\
742\end{array}$ \\
\hline 52 & $\begin{array}{l}\text { Lax } \\
\text { Medium } \\
\text { Strict }\end{array}$ & $\begin{array}{l}739 \\
636 \\
679\end{array}$ & $\begin{array}{l}814 \\
717 \\
762\end{array}$ & $\begin{array}{l}757 \\
646 \\
687\end{array}$ & $\begin{array}{l}787 \\
683 \\
728\end{array}$ & $\begin{array}{l}781 \\
647 \\
693\end{array}$ & $\begin{array}{l}782 \\
653 \\
701\end{array}$ \\
\hline 53 & $\begin{array}{l}\text { Lax } \\
\text { Medium }\end{array}$ & $\begin{array}{l}539 \\
518\end{array}$ & $\begin{array}{l}628 \\
777\end{array}$ & $\begin{array}{l}571 \\
547\end{array}$ & $\begin{array}{l}723 \\
754\end{array}$ & $\begin{array}{l}668 \\
660\end{array}$ & $\begin{array}{l}677 \\
679\end{array}$ \\
\hline
\end{tabular}




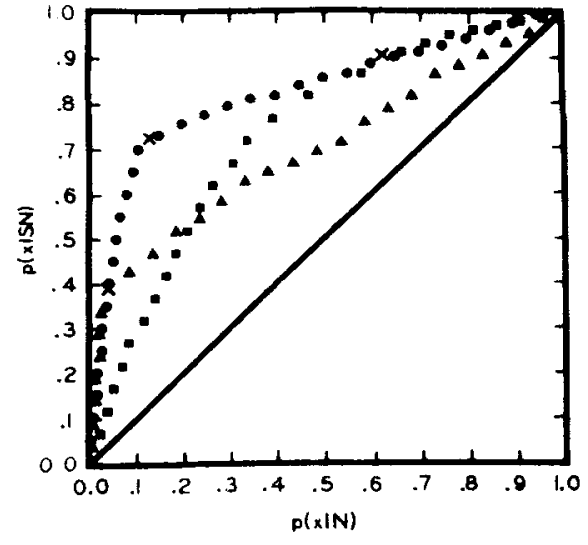

Fig. 5. ROCs obtained from S 1, Experiment 2. The $X s$ represent the yes-no operating points obtained with different response criteria. The triangles, circles, and squares are derived from response latencies obtained with strict, medium, and lax criteria, respectively.

hypothesis of Gescheider et al that the closer an observation is to the S's criterion, the greater the response latency will be. As the $S$ changes the location of his response criterion, an increase in the mean distance (from the criterion) of observations leading to the response which increases in frequency would be expected. (Interestingly enough, the validity of this argument depends upon the form of the underlying distributions assumed in the model. If other distributions are assumed instead of the normal distributions associated with TSD, then the average distance from the criterion of observations falling on one side of the criterion need not increase monotonically as the area under the density function on that side of the criterion increases.)

The data of $\mathbf{S} 2$ and $S 3$ are less orderly than those of $S 1$ with respect to criterion placement and do not in this regard provide support for the hypothesis of Gescheider et al. On the other hand, the fact that for all Ss, correct "yes" responses were made with shorter mean latency than incorrect "yes" responses, and correct "no" responses were made with shorter latency than incorrect "no" responses, does support this hypothesis. It is possible that $\mathrm{S} 1$ 's latencies were more orderly with respect to criterion placement than were those of $S 2$ and $S 3$ as a result of the fact that $S 1$ adopted more extreme criteria than did the other Ss. The differences in the criteria adopted by the Ss can be seen in Figs. 5-7. Since $S 2$ adopted the criteria in the order lax, strict, medium, it is also possible that S 2's latencies were decreasing as a result of general practice effects, although an attempt was made to provide enough practice to make such effects unlikely, and no general decrease in the latencies was noted across the five sessions in which data were obtained with a given criterion placement.

A method of deriving a ROC from yes-no response latencies was described in the discussion of Experiment 1. For each criterion adopted by each $\mathrm{S}$ in Experiment 2, ROCs were constructed in the same way and are shown in Figs. 5-7. The ROCs based upon latencies of responses made when the $S$ s maintained medium criteria are similar in general appearance to those from Experiment 1. As in the previous experiment, the segments of these ROCs which are based upon "yes" responses show somewhat more curvature than the segments based upon "no" responses. If these ROCs are plotted on normal-normal coordinates, they are fitted better by two straight line segments than by one, as is true of the ROCs of Experiment 1.

As can be seen in Figs. 5-7, when Ss adopted a strict criterion, the portion of the resulting ROC derived from the latencies of their "yes" responses was found to fall close to the ROC obtained with a medium criterion, while the portion derived from "no" responses lies noticeably below the ROC obtained with a medium criterion. Conversely, when Ss adopted a lax criterion the portion of the resulting $R O C$ which was based upon "no" responses was found to fall much closer to the medium-criterion ROC than the portion based upon "yes" responses. When the ROCs resulting from strict and lax criteria are plotted on normal-normal coordinates, they too are fitted better by two straight lines (one for the "yes" segment and one for the "no" segment) than by a single straight line. The fact that portions of latency ROCs change as Ss

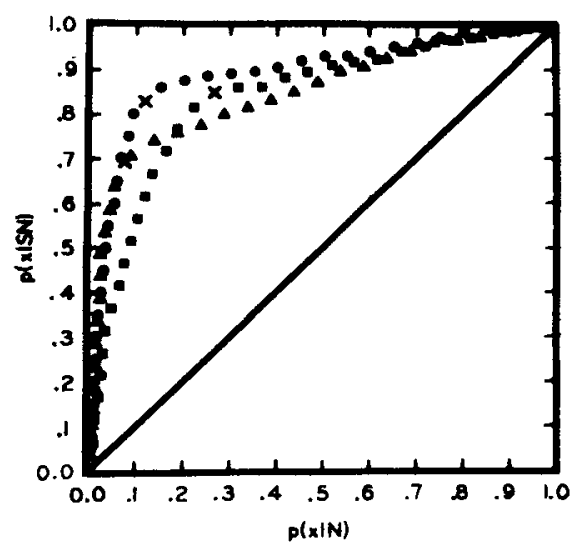

Fig. 6. ROCs obtained from S 2, Experiment 2. (See caption for Fig. 5.)

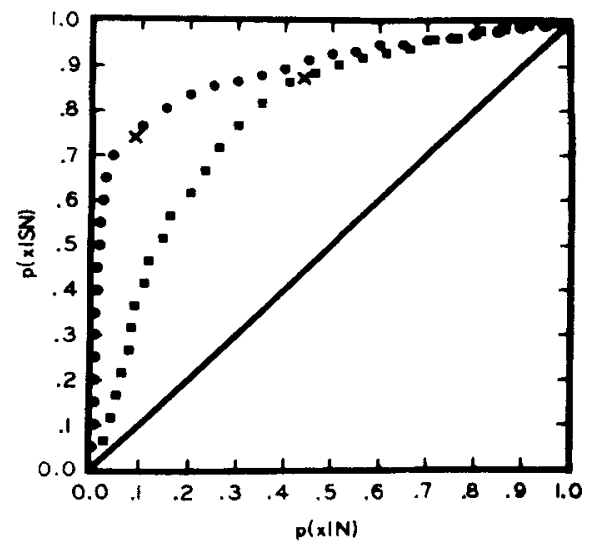

Fig. 7. ROCs obtained from 53 , Experiment 2. (See caption for Fig. 5.)

change their criteria means, of course, that these ROCs do not simply result from monotonic transformations of the same decision axis.

The $X$ symbols in Figs. 5-7 represent the proportions of "yes" responses given signal plotted against the proportions of "yes" responses given noise alone for different decision criteria. That is, these $\mathrm{Xs}$ are points on (yes-no) ROCs obtained by having Ss vary their decision criteria. As Norman and Wickelgren (1969) note, such yes-no points are constrained to fall upon the latency ROCs derived (in the manner described above) from the same "yes" and "no" responses. The yes-no points obtained with lax and strict response criteria do not have any such necessary relationship to the latency ROC derived from responses made with a medium response criterion, however.

\section{EXPERIMENT 3}

The third experiment was conducted for the same basic reasons as the first two: to learn more about the relations among confidence ratings, binary decisions, and response latency. As in the first two experiments, a comparison was made between results obtained with a yes-no procedure and results obtained with a confidence-rating procedure. Response latencies were collected under each procedure.

Method

Subjects. The Ss were four female graduate students at the Central Institute for the Deaf. All Ss had clinically normal hearing and none had previously served as $a$ S in any psychophysical experiment. The Ss were paid for their participation.

Apparatus. Acoustic stimuli were produced with standard laboratory components, including an oscillator, noise generator, electronic switch, and a bandpass filter. The presentation of 
signals, collection of responses, and measurement and recording of response latencies were handled for four $S s$ at a time by a small digital computer (Digital Equipment Corporation, Model PDP 8/S). The Ss were each seated in a separate sound-attenuating chamber. The stimuli were presented monaurally, to the Ss' right ears, using earphones (Telephonics, Model TDH-49).

Procedure. The Ss' task was to detect a $1,000-\mathrm{Hz}$ tone pulse, $148 \mathrm{msec}$ in duration, which was presented in a continuous background of band-limited $(100-3,000 \mathrm{~Hz})$ white noise. The noise spectrum level was 44.8 dB SPL, and the signals were presented with a value of $10 \log \left(\mathrm{E} / \mathrm{N}_{\mathrm{o}}\right)$ of 12.5 for all conditions, for all listeners. On each trial an observation light flashed on for $148 \mathrm{msec}$ and a tone pulse was presented during the light flash with a probability of 0.5 . A 1,500-msec response interval followed this observation interval. When a response was made during the response interval, a light came on and remained on until the end of the response interval to indicate to the $S$ that the response had been recorded. Without the Ss' knowledge, response latencies were recorded from the beginning of the observation intervals. Three seconds elapsed between the onsets of successive trials, and 100 trials constituted one 5-min listening block. Six or seven such blocks were run per hour, with several brief rest periods interspersed among the blocks.

The two response procedures used in Experiment 3 were the yes-no procedure described in Experiments 1 and 2, and an eight-category rating-scale procedure. The lights and sets of buttons used with these methods were mounted in the tops of boxes ( $2 \times 5 \times 7$ in.) placed in front of each S. Only one response procedure was used during any single listening block.

In the first five sessions of the experiment, the Ss were trained under the binary-decision procedure. They were encouraged to make "yes" and "no" responses equally often and, following each 100-trial block, they were told how many correct responses they had made. In Sessions 6-8 they were trained under the rating procedure, with responses on the right four keys (the rating boxes looked somewhat like a truncated piano keyboard) interpreted to mean, from right to left, (1) "very certain that a signal was presented," (2) "moderately certain that a signal was presented," (3) "possibly a signal was presented," and (4) "you made me guess, so I'll say that a signal was presented." The left four keys continued this scheme,

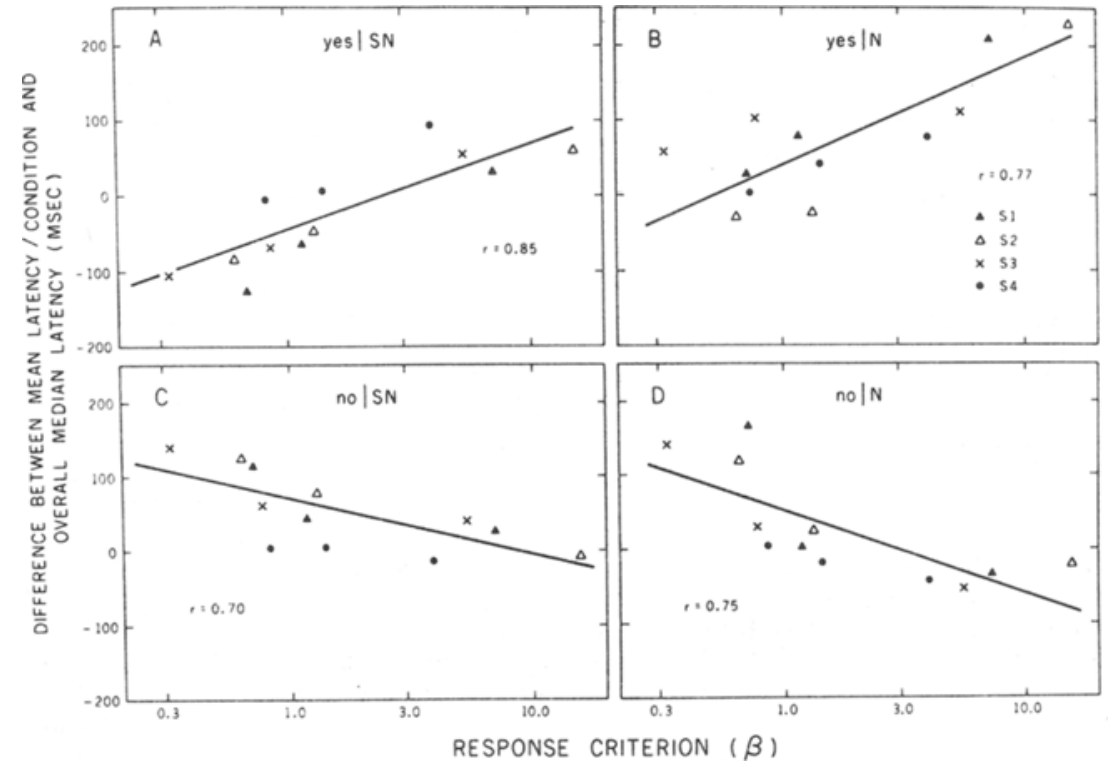

Fig. 8. Differences between the overall median latency and the mean latency for each experimental outcome (hits, false alarms, misses, and correct rejections) shown as a function of the response criterion, beta, for each $S$ in Experiment 3 . Higher values of beta correspond to stricter criteria for responding "yes, a signal was presented."

with responses on the leftmost key interpreted as "very certain that a signal was not presented." The Ss were encouraged to use all of the keys, but not necessarily equally often. The ninth session of the experiment was devoted to training the Ss in the use of the yes-no buttons on a separate response box, using the binary responses with either lax, medium, or strict criteria. The criteria were associated, through instructions, with the rating categories described above. Under the lax criterion they were told to make the response "yes" whenever they would have pressed one of the rating keys numbered " 1 " through " 6 ," had they been using the rating procedure. Under the medium criterion they were told to respond "yes" whenever they would have used keys " 1 " through " 4 ," and under the strict criterion they were only to respond "yes" when they would have used keys " 1 " or " 2 ." In each case the instructions for the "no" responses were the complement of those for the "yes" responses.

In Sessions 10-15 the Ss were run under the four conditions, rating, and strict, medium, and lax binary decisions, with the condition chosen randomly for each block of trials. At least seven blocks of trials were run for each $\mathbf{S}$ under each condition.

\section{Results and Discussion}

Figure 8 shows the difference between the mean latency for each of the experimental outcomes (hits, misses, false alarms, and correct rejections) under the binary-decision conditions and the overall median latency, for each $S$, plotted as a function of the criterion index, beta. ${ }^{1}$ (A discussion of beta may be found in Swets et al, 1961.) The three data points for each $S$ in a single panel of Fig. 8 correspond to the three criteria under which each $S$ was tested with the yes-no procedure. The latencies of "yes" responses tend to increase as the criterion is made more strict and latencies of "no" responses tend to increase as the criterion is made more lax, as predicted by the hypothesis of Gescheider et al.2 The correlations between the latency differences and log beta are similar in magnitude for the four experimental outcomes, ranging from 0.70 to 0.85 in absolute value. It can also be seen from the data in Fig. 8 that if the instructions to vary the criterion are not particularly effective for a given $S$, then that $S$ will not show a very great shift in response latency from one criterion to another. This may explain why S 2, in Experiment 2, did not show latency changes which were consistent with much of the data reported elsewhere in this article.

Figure 9 shows ROCs for each of the four conditions of Experiment 3. The larger symbols represent the yes-no operating points obtained with the three criteria, while the smaller symbols represent latency-based ROCs derived with the method described in the discussion of Experiment 1. The filled circles are ROCs for the confidence-rating condition. It is 


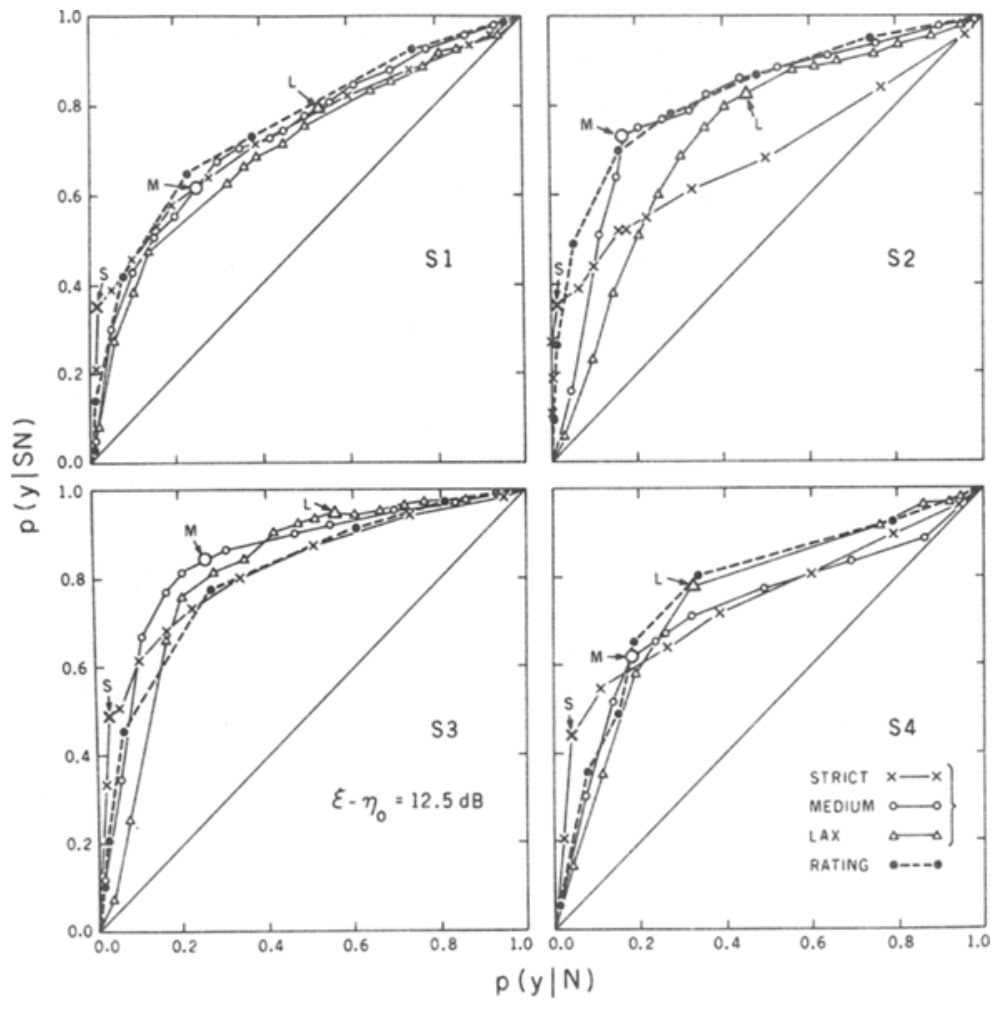

Fig. 9. ROCs for the data of Experiment 3. The solid circles represent rating-scale responses. The large circles, triangles, and $\mathrm{Xs}$, which are adjacent to the letters $S, M$, and $L$, are the binary-decision operating points for the three response criteria. The smaller circles, triangles, and $\mathrm{Xs}$ are points on latency-based ROCs for the three response criteria.

impossible to draw a simple generalization from these data. Very similar ROCs may be fitted to the data for ratings and for the latencies associated with each of the three criteria, for $\mathrm{S} 1$. On the other hand, S 2 shows rating and yes-no operating points which are quite similar, but her latency ROCs are only slightly more informative than straight lines connecting the yes-no operating point to the origin and to the point $(1,1)$. The latency ROCs for S 3 and S 4 are neither as close to being straight lines as are those of $\mathbf{s} 2$, nor as good predictors of the rating ROCs as are those of $S 1$. Evidently the relation between confidence and latency is fairly strong for some Ss but weak for others.

Another way in which ROCs derived from ratings and from the latencies of yes-no responses can be compared is by determining the areas under each. Green and Swets (1966, pp. 46-48) have shown that the area under the ROC, as a proportion of the unit square, is an estimate of the probability that a decision would be correct on the same sensory task if a two-alternative forced-choice procedure were used. The areas under each of the confidence-rating ROCs and latency-based ROCs were measured with a planimeter and are presented in Table 4 , along with the areas formed by connecting the binary-decision operating points to the origin and to the point $(1,1)$ by straight lines. Such straight-line ROCs represent the latency ROCs which would be produced if the latencies of yes and no responses did not depend on whether or not these responses were correct.

The differences between the areas under the straight-line ROCs and the areas under the latency ROCs are an indication of the amount of information gained if, in addition to knowing what response was made, the latency of the response is also considered. While the information gained by knowing the latency of responses clearly varies among the $\mathrm{Ss}$, the averages across Ss in Table 4 are probably representative for the particular procedures used in Experiment 3. Thus the smallest areas in the table are from the straight-line ROCs, latency-based ROCs yield somewhat larger areas, and the largest areas of all are obtained from confidence-rating ROCs. To a first approximation, Table 4 can be interpreted as follows: the amount of additional information gained by considering the response latency as well as the binary response itself is on the order of half that gained by moving from binary decisions to an 8-point rating scale. The agreement between latency-based and confidence-rating ROCs observed in Experiment 1 was, of course, closer than that observed in Experiment 3, even for latencies obtained with a medium response criterion in the latter experiment.

\section{SUMMARY AND CONCLUSIONS}

In three auditory detection experiments, the latencies of binary decisions tended to be longer for less frequent responses and for incorrect responses, than for more frequent responses and correct responses. In addition, the mean latency of binary responses was greater as Ss reported less certainty about the stimuli presented.

Changes in the Ss' response criteria produced by various instructions yielded changes in average response latencies which generally were consistent with the hypothesis suggested by Gescheider et al that the farther an observation falls from the current criterion, the shorter the latency of the resulting response. The data of the present experiments give only indirect support to that hypothesis, since they are based on the relations between average stimuli and average response latencies. If a given model of stimulus processing were assumed, more direct evidence could be obtained by measuring both stimuli and response latencies on a trial-by-trial basis. The fact that the forms of the latency-based ROCs varied systematically as the Ss varied

Table 4

Areas Under ROCs (Experiment 3)

\begin{tabular}{|c|c|c|c|c|c|c|c|}
\hline & \multirow[b]{2}{*}{$\begin{array}{c}\text { Rating } \\
\text { ROC }\end{array}$} & \multicolumn{2}{|c|}{ Strict Criterion } & \multicolumn{2}{|c|}{ Medium Criterion } & \multicolumn{2}{|c|}{ Lax Criterion } \\
\hline & & $\begin{array}{c}\text { Latency } \\
\text { ROC }\end{array}$ & $\begin{array}{l}\text { Straight- } \\
\text { line ROC }\end{array}$ & $\begin{array}{c}\text { Latency } \\
\text { ROC }\end{array}$ & $\begin{array}{l}\text { Straight- } \\
\text { line ROC }\end{array}$ & $\begin{array}{c}\text { Latency } \\
\text { ROC }\end{array}$ & $\begin{array}{l}\text { Straight- } \\
\text { line ROC }\end{array}$ \\
\hline $\begin{array}{ll}\text { S } & 1 \\
\text { S } & 2 \\
\text { S } & 3 \\
\text { S } & 4\end{array}$ & $\begin{array}{l}76.1 \\
82.6 \\
81.1 \\
76.1\end{array}$ & $\begin{array}{l}73.5 \\
68.6 \\
82.2 \\
73.1\end{array}$ & $\begin{array}{l}66.3 \\
66.7 \\
72.7 \\
69.3\end{array}$ & $\begin{array}{l}73.5 \\
79.5 \\
84.9 \\
72.0\end{array}$ & $\begin{array}{l}67.8 \\
77.3 \\
79.2 \\
71.2\end{array}$ & $\begin{array}{l}71.2 \\
73.1 \\
81.4 \\
74.2\end{array}$ & $\begin{array}{l}62.5 \\
68.2 \\
69.3 \\
72.0\end{array}$ \\
\hline Mean & 81.5 & 74.4 & 68.8 & 77.5 & 73.9 & 75.0 & 68.0 \\
\hline
\end{tabular}


their criteria makes it clear, however, that the hypothesis of Gescheider et al does not give a complete account of the factors determining response latencies in auditory signal detection.

In general, the present experiments lead to the conclusion that latency-based ROCs can differ considerably from those derived from binary decisions or confidence ratings. In some situations it is unlikely that latency-based ROCs will diverge very greatly from binary-decision or rating ROCs, and then these three types of ROC can appear to be quite similar. This is the case for (1) medium-criteria and medium-to-low values of detectability or very high values of detectability, (2) the segment of the ROC below the binary-decision operating point for strict criteria, and (3) the segment above the binary-decision operating point for lax criteria. However, it is clear that there is an additional source of variability (or "noise") in response latencies, as compared to confidence ratings, and that this variability can be reflected in large differences between latency-based ROCs and those based on binary decisions or confidence ratings when these types of ROC are not constrained to fall close to each other.

Thus latency-based ROCs should probably not be viewed as a prime source of information about sensory processing alone. Response latencies are known to be influenced by many factors, and this is undoubtedly also the case for latency-based ROCs. Yager and Duncan (1971) reach a sirnilar conclusion in a study in which ROCs are derived from the response latencies of goldfish given a generalization task.

Norman and Wickelgren (1969) present latency-based ROCs obtained in an experiment concerned with short-term memory which are very similar in form to those presented in Fig. 4. The fact that ROCs of similar form are obtained in a number of experiments in which the stimuli, the tasks, and the response procedures differ considerably, suggests that there are similarities in the processes employed by the Ss in these different experiments. Perhaps the use of response latencies can be helpful in the attempt to discover the similarities and differences of the processing employed by the Ss in these diverse situations.

\section{REFERENCES}

CARTERETTE, E. C., FRIEDMAN, M. P.. \& COSMIDES. R. Reaction-time distributions in the detection of weak signals in noise. Journal of the Acoustical Society of America, 1965, 38, 531-542.

CARTWRIGHT, D. The relation of decision-time to the categories of response. American Journal of Psychology, 1941,54,174-196.

EGAN, J. $\dot{P} .$, SCHULMAN, A. I., \& GREEN BERG, G. Z. Operating characteristics determined by binary decisions and by ratings. Journal of the Acoustical Society of America, 1959, 31, 768-773.

EMM ERICH, D. S. Receiver-operating characteristics determined under several interaural conditions of listening. Journal of the Acoustical Society of America, $1968,43,298-307$.

FER NBER GER, S. W., GLASS, E. HOFFMAN, I., WILLIG, M. Judgment times of different psychophysical categories. Journal of Experimental Psychology, 1934, 17, 286-293.

FESTINGER, L. Studies in decision: $I$, Decision-time, relative frequency of judgment and subjective confidence as related to physical stimulus difference. Journal of Experimental Psychology, 1943a, 32, 291-306.

FESTINGER, L. Studies in decision: II. An empirical test of a quantitative theory of decision. Journal of Experimental Psychology, 1943b, 32, 411-423.

GESCHEIDER, G. A., WRIGHT, J. H., WEBER, B. J., KIRCHNER, B. M., \& MILLIGAN, E. A. Reaction time as a function of the intensity and probability of occurrence of vibrotactile signals. Perception \& Psychophysics, 1969, 5, 18-20.

GREEN, D. M., \& SWETS, J. A. Signal detection theory and psychophysics. New York: Wiley, 1966.

JOHNSON, D, M. Confidence and speed in the two-category judgment. Archives of Psychology, Columbia University, 1939, No. 241.

KATZ, L. A comparison of type II operating characteristics derived from confidence ratings and from latencies. Perception \& Psychophysics, 1970, 8, 65-68.

MEDIN, D. L., BORKHIUS, M. L., \& DAVÍs, R. T. Response latency and brightness judgments by monkeys. Journal of Experimental Psychology, $1970,83,480-485$.

NORMAN, D. A., \& WICKELGREN, W. A. Strength theory of decision rules and latency in retrieval from short-term memory. Journal of Mathematical Psychology, 1969, 6, 192-208.

SWETS, J. A., TANNER, W. P., JR., \& BIRDSALL, T. G. Decision processes in perception. Psychological Review, 1961. $68,301-340$.

WATSON, C. S., RILLING, M. E., \& BOURBON, $W$. T. Receiver-operating characteristics determined by a mechanical analog to the rating scale. Joumal of the Acoustical Society of America, 1964, 36, 283-288.

WOODWORTH, R. S., \& SCHLOSBERG, $H$. Experimental psvchology. New York: Holt, 1954. Pp. 1-42.

Y A G ER , D., \& DUNCA N, I. Signal-detection analysis of luminance generalization in goldfish using latency as a graded response measure. Perception \& Psychophysics, 1971, 9, 353-355.

\section{NOTES}

1. Beta may be computed by dividing the ordinate of the standard normal curve with area $p$ (yes | SN) to the right of it by the corresponding ordinate with p(yes |N) to the right of it.

2. Response latencies did not vary as much across rating responses as they did when yes-no criteria were manipulated. Latencies for ratings were longest toward the center of the rating scale, shortest at the "signal" end. and intermediate at the "noise" end. Likelihood ratios [ISN/N $(x)]$ were calculated for the rating critena to either side of the longest latency rating responses, and for three of the $S$ s these values of $1(x)$ were close to 1.0 . For $S 3$ the ratings with longest latency were given when $l(x)=0.26$, a very lax criterion. To extend $l(x)=0.26$, a very lax criterion. To extend
the hypothesis of Gescheider et al to confidence ratings, one may interpret rating latencies as indicating a "second order" confidence judgment. Thus the rating latency might be a measure of the $S$ 's certainty that a particular rating is appropriate to a given stimulus rather than the immediately higher or lower rating. Large within-rating variance in latencies, as compared to between-rating variance, in the data of Experiment 3 gave support to this hypothesis.

(Received for publication May 18, 1971.) 